\title{
Learning effective amino acid interactions through iterative stochastic techniques
}

\author{
Cristian Micheletti ${ }^{1}$, Flavio Seno ${ }^{2}$, Jayanth R. Banavar ${ }^{3}$ and Amos Maritan ${ }^{1}$ \\ (1) International School for Advanced Studies and INFM, Via Beirut 2, 34014 Trieste, Italy \\ (2) INFM -Dipartimento "G. Galilei" , Via Marzolo, 8 Padova, Italy \\ (3) 104 Davey Laboratory, The Pennsylvania State University, University Park, Pennsylvania 16802
}

(October 30, 2018)

\begin{abstract}
The prediction of the three-dimensional structures of the native state of proteins from the sequences of their amino acids is one of the most important challenges in molecular biology. An essential ingredient to solve this problem within coarse-grained models is the task of deducing effective interaction potentials between the amino acids. Over the years several techniques have been developed to extract potentials that are able to discriminate satisfactorily between the native and non-native folds of a pre-assigned protein sequence. In general, when these potentials are used in actual dynamical folding simulations, they lead to a drift of the native structure outside the quasinative basin. In this study, we present and validate an approach to overcome this difficulty. By exploiting several numerical and analytical tools we set up a rigorous iterative scheme to extract potentials satisfying a pre-requisite of any viable potential: the stabilization of proteins within their native basin (less than 3-4 $\AA$ cRMS). The scheme is flexible and is demonstrated to be applicable to a variety of parametrizations of the energy function and provides, in each case, the optimal potentials.
\end{abstract}

\section{INTRODUCTION}

\section{A. Background}

In recent years, there have been numperqus studies pertaining to the determination of effective amino acid interactions for coarse-grained models of proteinst 16 . The reasons are two-fold. If reliable interaction potentials were available, they could be used, in principle, to predict the native state of a known protein sequence through energy minimization techniques - a possibility underscored by the experimental observation of Anfinsent7 that proteins fold rapidly and reversibly into a unique conformation which he postulated to be the free energy minimum. Second, the knowledge of good potentials is a necessary ingredient to perform_design of protein-like sequences, i.e. finding the sequence which has the assigned target structure as its native state 129.

A simple and pepular method for the extraction of the interaction potentials is the so-called quasi-chemical approximation $61,11,12$, which infers the strength of pairwise interactions from the relative abundance of distinct pairs of amino acids in contact. In favour of this technique are its simplicity of implementation and robustness against use of different sets of proteins used to extract the data. The quasichemical approach is, however, an approximate scheme since it neglects the peptide bonding of the amino acids bytregting them as a gas. In this respect it can be viewed as a first correction to a sort of mean-field approximation $10,14,5,30,31$. Quasi-chemical methods have proved valuable since they can score satisfactorily as far as thermodynamic stability is concerned. In fact, they usually assign low energies to the native state of a sequence compared to the mean energy of the same sequence mounted on unrelated structures of the same length.

Other alternative extraction strategies 1 1 , 2, 10 , 31 pioneered by Crippen 1 use the thermodynamic stability criterion as the extraction method itself rather than as a mere validating tool. These schemes aim at finding a set of potentials so that given a protein sequence, its native state is recognized as having an energy well-below other conformations of the same length (decoys). A major advantage of such schemes is the possibility 31 of verifying directly whether the chosen parametrization of the free energy is appropriate. In fact, if the energy function introduced to describe the system is too simplistic, then it will not be possible to adjust its parameters so that the native states of all the proteins have lower energy than each of the competing decoy conformations. In fact, recent studies 32 have pointed out that a pairwise energy function may not stabilize even a single protein such as crambin. There are at least two explanations for the failure to learn good sets of potentials: one possibility is that structures too similar to the native one have been included in the training set of decoy conformations. The other, more serious concern, is that the parametrization for the energy function is too simple to capture the physics of the problem. Therefore, a given set of proteins might 
be unlearnable with a particular energy function (and strictly speaking might remain unlearnable with any energy function if infinite precision is required).

A key difficulty in implementing this powerful procedure is in the careful choice/generation of the decoy structures. In many instances, the decoys are generated by taking compact "chunks" of suitable length from a bank of proteins (threading) 33. Such decoys may not be physical for certain sequences because of steric constraints and are usually not very stringent, i.e. they do not compete significantly with the native state to be occupied below the folding transition temperature. This ends up with placing rather loose or unphysical constraints on the extracted potentials.

Both the potentials extracted mith the latter approach or the quasi-chemical one, undoubtedly capture the main features of amino acid chemistry 34 and of the folding process 30 . However, they have a range of applicability limited within the same scheme that was used for extraction. For example, the performance of the potentials determined using the quasi-chemical method is unsatisfactory when they are applied to unbiased folding simulations. In particular, dynamical trajectories starting from a protein's native state well below the folding transition temperature always escape from the quasi-native basin (with a 3-4 A root mean square deviation per site35).

An alternative strategy 36 would be to start from a trial set of potentials, carry out repeated folding processes with the aid of a computer and find the structures with the lowest energy. If any of these structures are significantly different from the native ones, then the potentials are modified so that their energy is increased above the target native state (destabilization). This process can be repeated until the native state is fully stabilized. More generally, the process should be carried out simultaneously for a set of non-homologous proteins. A key difficulty of the strategy is the need to have an efficient folding algorithm, which, in principle, could lead to the folding of a given sequence from the denatured state into its native state conformation corresponding to a given set of interaction parameters. In other words, if one has a powerful folding alogorithm, one can tune the parameters of the potential just right to ensure that the native states of a set of proteins are accurately learnt.

\section{B. A new iterative strategy}

Here, we will present a general strategy for determining the effective interactions between the coarse-grained degrees of freedom of a protein. Our procedure does not entail the difficulties associated with the methods described above. The key idea is the observation that the native states of protein must at least satisfy the Anfisenian requirement of being located at the bottom of a smooth free energy minimum 3738 with a wide basin of attraction 39 This suggests a straightforward approach:

1. Begin with an initial guess of the potential parameters.

2. Start from the native states of several proteins and carry out an unbiased Monte Carlo (or molecular dynamics) simulation (say at zero temperature) and determine several accessible local minima for each of the proteins.

3. Modify the potential parameters in such a way as to destabilize these conformations in favor of the known native state conformations.

4. Iterate this procedure by returning to (2).

After several iterations, one would expect to converge to a set of potential parameters which best capture the optimal shape of the free energy landscape in the vicinity of all the native state structures.

Our method adopts the thermodynamic stability scheme described before but with the proviso that the decoys would be generated by an explicit and simple dynamical process. The structures so generated are guaranteed to be stringent competitors of the native structure in housing the sequence belpw the folding transition temperature.

Our strategy is a basic pre-requisite for viable effective potentials 35.40 and hence is an obligatory step along the difficult route to fully-automated folding prediction. Since the scheme is both flexible and optimal, it can be used to compare the performance of many different scoring functions or parametrizations and hence select the most promising one for ab initio folding simulations.

A fundamental question pertaining to our new strategy is "How well can the native state be stabilized for a given form of the energy function?". In this article, we will tackle this question and determine the optimal choice of the parameters for a given energy function in order to approach the native states as well as possible.

We proceed by considering a set of 20 single chain proteins (training set) taken from different protein families and introduce a pairwise energy function to describe the interactions between the amino acids. Then, by using an iterative procedure, we systematically modify the parameters characterizing the pairwise potentials to optimize the local stability of the native states. It is shown that the iterative procedure brings a systematic improvement of the quality of the potentials. The optimal potentials confer significant thermal stability . Moreover we check the quality 
of our potentials by testing them on sets of hundred of decoys believed to be very stringent, obtained by Levitt for seven heavily investigated proteins 11 . The results of the tests are found to be very encouraging.

The main message of our paper is to present and demonstrate the viability of the idea that the effective potential between amino acids can be learned by ensuring the local stability of the native states of many proteins simultaneously. There are obviously many ways of implementing this idea and we will present a few schemes here that we have tried and which yield remarkably good results.

\section{THEORY}

\section{A. The model}

Microscopic molecular dynamics techniques using presently available computational resources can follow the dynamics of short peptides for time scales significantly shorter than typical folding times. For these reasons, it has become customary to simplify both the representation of protein conformations and their dynamical behaviourt 4 . Most of the procedures adopted to coarse grain the microscopic degrees of freedom of proteins substitute a whole amino acid with an effective centroid placed at a suitable point along the CA-CB direction, or coinciding with one of these two atoms. This choice, that we shall also adopt in this study brings about a drastic simplification of the structure of the energy function involving only interactions between the centroids. The coarse-graining procedure that we adopted is inspired by the XFCC model of Covell and Jernigan 13 . According to this model, both the dihedral angles and peptide-bond length are discretized so that there are several degrees of freedom per amino acid (identified with CA atoms). This framework allows a faithful representation of protein backbones, since the coarse grained CA positions are typically within $1 \AA$ of the crystallographic positions. The preservation of the typical angles is carried out at the expense of variations in the peptide bond length, $d$, (the separation between consecutive CAs) which can be stretched within the bounds $2.6 \stackrel{\circ}{A}<d<4.7 \AA$. Within such a range of $d$, there are up to 40 possibilities for placing a CA centroid. This makes the Covell-Jernigan model probably the best compromise between having a description as close as possible to the continuum while retaining only few degrees of freedom 4 . Besides the CA's, we also introduce $\mathrm{CB}$ atoms constructed using a geometric rule (inspired from one obtained in the continuum 44 ) deduced from peptide geometry in the XFCC context:

$$
\vec{r}_{i}^{C B}=l(\hat{a} \cdot \cos \theta+\hat{b} \cdot \sin \theta)
$$

where:

$$
\hat{a}=\frac{\hat{s}_{i, i-1}+\hat{s}_{i, i+1}}{\left|\hat{s}_{i, i-1}+\hat{s}_{i, i+1}\right|} \quad \hat{b}=\frac{\hat{s}_{i, i-1} \wedge s_{i, i+1}}{\left|\hat{s}_{i, i-1} \wedge \hat{s}_{i, i+1}\right|}
$$

In the previous equations, $\hat{s}_{i, j}$ is the unit vector:

$$
\hat{s}_{i, j}=\frac{{\overrightarrow{r_{i}}}^{C A}-{\overrightarrow{r_{j}}}^{C A}}{\left|{\overrightarrow{r_{i}}}^{C A}-{\overrightarrow{r_{j}}}^{C A}\right|}
$$

while $l$ is the distance of the $\mathrm{CB}$ atom from the $\mathrm{CA}$ atom that we choose equal to $3 \AA$, $\theta$ is an appropriate angle optimally chosen to be $37.6^{0}$ and $\vec{r}_{k}^{C A(C B)}$ is the position of the $k$-th $\mathrm{CA}(\mathrm{CB})$ atom along the chain. When crystallographic positions are used, such restrictions place the $\mathrm{CB}$ within $0.3 \AA$ of the true position; when discretized values for $\mathrm{CA}$ locations are used, the discrepancy is increased to about $1 \AA$.

\section{B. Parametrization of potential energies of interaction}

Most of the results, that we present here, are for a simple energy function with interactions only between pairs of non-consecutive CA atoms,

$$
\mathcal{H}=\sum \Delta\left(\left|\vec{r}_{i}^{C A}-\vec{r}_{j}^{C A}\right|\right) \cdot \epsilon\left(S_{i}, S_{j}\right)+10 \cdot \epsilon_{r} \cdot\left[\left(\frac{4.65}{\left(\left|\vec{r}_{i}^{C A}-\vec{r}_{j}^{C A}\right|\right)}\right)^{2}-1\right] \cdot \Theta\left(4.65-\left|\vec{r}_{i}^{C A}-\vec{r}_{j}^{C A}\right|\right)
$$

where, 


$$
\Delta(r)=\frac{1}{2}+\frac{1}{2} \tanh \frac{6.5-r}{2}
$$

and $\Theta$ is the step function. We will refer to this model as the model A.

$\Delta$ denotes the distance-dependent strength of interactions between the $i$-th and the $j$-th amino acids along the sequence mounted on the structure $\Gamma, \epsilon$ is the interaction matrix and $S_{i}$ denotes the type of the $i$ th amino acid in the sequence. $\epsilon_{r}$ is a repulsive term that penalizes cases where two non-consecutive pairs of CA's are closer than $4.65 \AA$, a circumstance rarely encountered in protein structures. Altogether, there are 211 parameters to be learnt (with all of them eventaully scaled by the normalization condition).

\section{Monte Carlo dynamics}

The dynamics in conformation space is carried out using a Monte Carlo technique. A newly generated conformation is accepted according to the standard Metropolis rule. At each attempted MC step, we move up to 2 of consecutive protein residues to unoccupied discrete positions. The new positions are constrained to satisfy a set of suitable physical constraints that we deduced by a statistical analysis of the CA and CB positions of an ensemble of over hundred single-chain globular proteins within the XFCC model. More precisely:

- The separation $d$ between two consecutive $C A$ atoms (measured in $\AA$ ) must remain in the range $2.6<d<4.7$;

- Two non-consecutive $C B$ atoms must not be closer than $2 \AA$.

- Two non-consecutive $C A$ and $C B$ atoms must not be closer than $2 \AA$.

- The chain length is allowed to fluctuate by up to a maximum value of $4 \AA$ (in magnitude) with respect to the original length.

The Monte Carlo algorithm was used to relax the crystallographic structure (XFCC-discretized) to its lowest energy states. This is conveniently done by setting the MC temperature close to zero and carrying out typically 300,000 attempted moves. The relaxed configurations are used as decoy structures for refining the potentials by requiring that the native state has lower energy than the relaxed ones. This amounts to a requirement that the optimal potentials should, at least, ensure the best local stability for the protein.

\section{Finding the optimal potentials}

A convenient way to find the optimal potentials is the use of the perceptron algorithm 15 for the optimization of a set of linear inequalities.

In our case, the inequalities are of the form

$$
\mathcal{H}(S, \Gamma)-\mathcal{H}\left(S, \Gamma_{\text {decoy }}\right)<0
$$

and it is possible to find one such inequality for each decoy. Expression (6) can be rewritten as

$$
\begin{aligned}
\sum_{i>j=1}^{20}\left(n_{i j}^{d}-n_{i j}^{n}\right) \epsilon(i, j)+\epsilon_{r}\left(n_{r}^{d}-n_{r}^{n}\right) & \equiv \sum_{i>j=1}^{20} a_{i j}\left(\Gamma_{\text {decoy }}\right) \cdot \epsilon(i, j)+a_{r} \cdot \epsilon_{r} \\
& \equiv \mathcal{Q}\left(\Gamma_{\text {decoy }}, \epsilon\right)>0
\end{aligned}
$$

where $n_{i j}^{n, d}$ denotes the number of native/decoy contacts involving amino acids of types $i$ and $j$ and $n_{r}^{n, d}$ denotes the strength of the native/decoy repulsive term. Given the native state $\Gamma$ and the sequence $S$, the 211 entries of $a_{i, j}\left(\Gamma_{\text {decoy }}\right)$ plus $a_{r}$ depend only on the geometrical properties of the decoy structure.

For a given set of $M$ inequalities to be satisfied simultaneously, it is convenient to identify the one (denoted with l) that, with the trial potentials is the worst satisfied one:

$$
\mathcal{Q}\left(\Gamma_{l}, \epsilon\right)<\mathcal{Q}\left(\Gamma_{k}, \epsilon\right) \quad k=1, \ldots, M, \quad k \neq m
$$

The selection of the conformation $l$ can be done both when $\mathcal{Q}\left(\Gamma_{l}, \epsilon\right)$ is negative (not all the inequalities are satisfied) and when it is positive (all the inequalities are already satisfied). $\mathcal{Q}\left(\Gamma_{l}, \epsilon\right)$ is called the stability of the set of inequalities for a given choice of $\epsilon$. 
Once $l$ has been determined, one updates the trial potentials, $\epsilon(i, j)$ (or $\epsilon_{r}$ ) by adding a quantity proportional to $\left(a_{i j}\left(\Gamma_{l}\right)\right)$ (or $a_{r}\left(\Gamma_{l}\right)$, where the proportionality constant is chosen to be much smaller than 1 . With this new choice of the potentials, each inequality is re-valuated and the updating cycle is repeated. This method can be shown to converge to the optimal solution: the stability $\mathcal{Q}$ reaches a constant value (optimal stability) 45 , which can be of either sign. If it is negative, it means that no set of potentials can be found that consistently satisfies all inequalities in the set (unlearnable problem). To speed up the convergence process towards the optimal potentials, we found it useful to introduce an additional type of inequality besides (6), namely

$$
H\left(S_{i}, \Gamma_{i}\right)<0 \text {. }
$$

Such an inequality is useful for ensuring that the native state of the protein is stable against generally open conformations (with energy approximately zero) while the previously introduced inequalities required stability against competing decoys. The inequalities as in Eq. (10) provide stringent limitations to the parameter space, thus aiding the search for optimal parameters.

Intuitively, if the number of inequalities exceeds the number of parameters (211 in our case) and if the energy function is too simplistic it is unlikely that a solution will be found. Of course, correlations in the inequalities can make the problem unlearnable even with very few inequalities or learnable with many of them.

In any case, the stability threshold cannot increase upon enlarging the set of decoys/inequalities. As an example we show in Fig. 1 the behaviour of the stability as a function of the number of decoys obtained through relaxation with the same set of interaction potentials. It can be seen that due to correlations among the decoys, the stability does not decrease appreciably after a few dozens of them have been introduced. The results of Fig. 1 1 are valuable because they give us an estimate of the number $(30-50)$ of representative decoys to be collected at each iteration step of the potential update.

By updating the potentials, one can generate a set of decoys that are much closer in root mean square deviation (RMSD) to the target one than the previous decoys. This is seen in Fig. 11 where the abrupt decrease of stability is visible upon addition of the new decoys. The iterative potential update can be repeated until the stability becomes negative or even beyond that. At each iteration step, it is useful to monitor the average RMSD of the decoys from the native conformations. It can be anticipated that, as the iterations proceed, the RMSD will decrease down to a minimal value and then rise again. We consider this as a natural cutoff value - any decoy with a RMSD below this value ought to be identified with the native state itself for a given choice of the energy function. Of course, the better the choice of the energy functional, the lower this cutoff will be.

In our study, we have first applied this method to a single protein for didactic purposes. There after, we considered a set of 20 proteins (trial set) as representatives of the main folds (see table II) and attempted to learn them simultaneously by storing 100 decoys ( 5 for each them) before each potential update.

\section{RESULTS AND DISCUSSION}

We began by considering a single protein, PDB code: $1 \mathrm{vcc}$, which has 77 residues. Starting from a set of random potentials, we generated 30 decoys for which we computed the average RMSD, $\bar{g}$, from the native structure and its variance $\Delta \bar{g}$. With these decoys, we found the potentials by applying the perceptron algorithm and the normalization condition $\left(\sum \epsilon_{i, j}^{2}+\epsilon_{r}^{2}=1\right)$ which sets the energy scale. With the new interaction parameters, we generated 30 more decoys and kept repeating this procedure.

In Fig. 2 we show the RMSD, $\bar{g}$, as a function of the number of iterations. It is remarkable that, with such a simple model, $\bar{g}$ can be decreased dramatically from the initial value of about 6 Åto around 1.5-2.0 $\AA$, which is just over the order of the experimental uncertainty! This provides a nice demonstration of the fact that although, strictly speaking, the problem of learning the pairwise interactions is unlearnable 32 - for otherwise one would reach zero RMSD - it is nevertheless possible to stabilize the native state in the native basin within a low uncertainty. The decrease of perceptron stability as a function of iteration is shown in Figure 3.

It is instructive to analyze in detail the plot of Fig. 2 in order to clarify some issues in the use of the perceptron learning procedure. At the first iteration, we set the short range repulsive potential parameter $\epsilon_{r}$ equal to zero and select random values for the 210 interaction parameters. With this choice, very compact conformations can be reached and consequently the radius of gyration of the decoys is very small and the RMSD quite high (see Fig. 2). However, learning the potentials from this set of decoys leads to a strictly positive value for $\epsilon_{r}$. Indeed, starting from the second iteration, the radius of gyration approaches that of the native state and consequently the values of RMSD start to decrease systematically. Notably, just a few iterations are sufficient to set the correct relative scale between the pair potential interactions and $\epsilon_{r}$. This scale, which impacts on the overall compactness, and is analogous to the average 
value of the pairwise interaction cannot be unambiguously determined within other schemes for potential extraction such as the quasi-chemical approximation or threading.

Next, we attempted a task considerably more difficult, which was an attempt to stabilize the native states of twenty proteins simultaneously. The twenty proteins, shown in Table 1 were chosen among a list of non-redundant representatives of the main protein folds. At each iteration step, we generated 5 decoys for each of them. We saw an improvement as the iterations went on, although not as pronounced as for the single 1vcc protein with $\bar{g}$ decreasing to a value of $3.8 \pm 0.5 \AA$. The typical behavior is illustrated by that of a single protein (chosen to be 1vcc) during the learning procedure of the potential for the full set of 20 proteins. (see Fig. 面). The table of the extracted potential parameters is given in Appendix A.

As recommended by Lazaridis and Karplus 16 in their recent review article on potential extraction, as an independent test of the quality of our potentials we assessed their performance on a set of seven proteins (see first column of Table II) unrelated to those used for extracting the potentials and for which more than 600 stringent decoys structures (for each protein) have been derived 44 . This unbiased study ought to reflect the portability of our potentials, i.e. their applicability in contexts different from which they have been derived.

The test we have performed is the following. For each protein we compute the energy of the the native state $E_{g}$ and the energy of all the decoys $E_{i}(i=1, \ldots, M)$ (where $\mathrm{M}$ is the number of decoys for each single protein) by using our optimal potentials. With the correct potentials, $E_{g}$ should be always lower than any other $E_{i}$. In table [I] we report the ranking in energy of the ground state with respect to all the other decoys. The native state structure is never the highest ranking one but is always among the best 5 to $10 \%$. To better elucidate the quality of our potentials, we have created a scatter plot (Fig. 5) of the energy of the decoys (relative to the native state energy) versus the rmsd from the native state. From (Fig. 5), the two quantities are seen to be correlated. This is a highly non-trivial result since it is generally difficult to get such correlations even employing specially designed energy scoring functions 44 .

We now turn to a verification of how our potential compares with others in stabilizing the native state. To do this we decided to estimate a new set of potentials by applying the perceptron learning scheme to the decoys of Park and Levitt. In other words, we identify the set of potentials which maximally stabilize the ground state of the seven proteins chosen in ref. 44 with respect to their own competing decoys. With the new potentials we checked the asymptotic RMSD reached on each of the 20 proteins in our training set and compared it to the stability obtained with our previous optimal potentials. The results are reported in Fig. 6 We repeated the same analysis but working with the seven proteins of the Levitt data bank and the results are reported in Fig. 7. Remarkably, on the average, the optimal potentials are able to stabilize the proteins with an accuracy higher than those obtained by using the potentials extracted using the decoys of ref. 44.

As another stringent test, we compared the performance of our potentials with those extracted with the quasichemical approximation. According to the basic prescription of such a scheme, the strength, $\epsilon_{i j}$, of the interaction between two amino acid types $i$ and $j$ can be deduced from the number of contacting $i-j$ pairs, $f_{i j}$, and the relative abundance of the types, $n_{i}$ and $n_{j}$ in the proteins in the training set. Such potentials are determined up to a multiplicative constant and an additive one. We chose the additive constant of the quasi-chemical potentials by setting their average to zero. Finally we set the norm of the potential vector to 1 , as for the optimal set. These choices ensure that the two potentials sets are similar and can be compared on an equal footing. We also checked the robustness of the extracted potentials by checking that using ten additional proteins for the extraction procedure did not alter the potential values appreciably.

$$
\epsilon_{i j}=\log \frac{f_{i j}}{\left(2-\delta_{i j}\right) n_{i} \cdot n_{j}}
$$

Likewise, the strength of the repulsive term, $\epsilon_{r}$, was obtained by replacing, in equation (11), $f_{i j}$ with the number of non-consecutive CA pairs that are below $4.5 \AA$, while $n_{i, j}$ is replaced by the total number of CA atoms.

We have extracted the quasi-chemical potentials from the twenty proteins in the training set. Using this potential, and our Monte-Carlo procedure, we assessed how well the twenty proteins could be stabilized. The results are reported in Fig. 8. The figure shows that quasichemical potentials do not provide the best stability and their performance is somewhat worse than that obtained on learning with the Park and Levitt decoys.

As a final test, in order to verify the possibility of improving our approach, we have introduced a slightly more sophisticated model, model B, where we consider interactions between all possible pairs of CA and CB at a sequence separation greater than 1 . The specific interaction is concentrated on the $\mathrm{CB}$ atoms, when present. On the other hand, the interaction between CA atoms is assumed to be independent of the type of amino acids. Since glycine lacks a CB atom, a Gly pair will only interact through the CA-CA potentials. For this reason the CA-CA interaction can be identified in our model with the Gly-Gly interaction, while interactions between CA-CB will be of type Gly-X, where $\mathrm{X}$ is the amino acid type to which the $\mathrm{CB}$ belongs 4 . In the model, a short range repulsive energy is now present between all pairs of residues (CA-CA, CA-CB and CB-CB) and therefore the hard core repulsion described earlier is no longer needed. 
Using this second model, we repeated the same analysis as before and as demonstrated in Figures 9 and 10 and in Table III, we get an improvement of the results. This example is helpful in illustrating the possibility of using our novel optimization technique in selecting the "most physical" energy parameterization.

\section{CONCLUSIONS}

We have demonstrated how one may extract effective interaction potentials between amino acids in a coarse-grained description of a protein. The method relies on the possibility of finding a set of competitive decoys of the native state. We outlined an iterative procedure to generate these decoys which attempts to stabilize, at least locally, the native state. The results obtained with simple forms of the energy function are very promising - we were able to stabilize a set of 20 proteins to an average distance of less than 4 Aand moreover, the potentials, when applied to other test with completely unrelated decoys, yield encouraging results. The use of slightly more sophisticated and complete forms of the energy function together with a non-discretized representation of the protein should lead to even further improvement.

\section{ACKNOWLEDGMENTS}

This work was supported by grants from INFM and MURST-COFIN99.

${ }^{1}$ Crippen, G.M. Prediction of protein folding from amino acid sequence over discrete conformation space, Biochemistry, 30: 4232-4237, 1991.

${ }^{2}$ Crippen, G.M. Failures of inverse folding and threading with gapped alignement, Protein: Struct. Funct. Genet., 26: 167$171,1996$.

${ }^{3}$ Friederichs, M.S. and Wolynes, P.G. Toward protein tertiary structure recognition by means of associative memory hamiltonians, Science 246: 371-373, 1989.

${ }^{4}$ Maiorov, V.N. and Crippen, G.M. Contact potential that recognizes the correct folding of globular proteins, J. Mol. Biol., 227:876-888, 1992.

${ }^{5}$ Dima, R., Settanni, G., Micheletti C., Banavar, J. R., Maritan, A. Extraction of interaction potentials between amino acids from native protein structures. J. Chem. Phys. 112:9151-9166, 2000.

${ }^{6}$ Miyazawa, S. and Jernigan, R.L. Estimation of effective interreside contact energies from protein crystal structures: quasichemical approximation, Macromolecules 18: 534-552, 1985.

${ }^{7}$ Miyazawa, S. and Jernigan, R.L. Residue-residue potentials with a favorable contact pair term an unfavorable high packing density term, for simulation and threading, J. Mol. Biol., 256: 623-644, 1996.

${ }^{8}$ Mirny, L.A. and Shakhnovich, E.I. How to derive a protein folding potential? A new approach to an old problem, J. Mol. Biol., 264: 1164-1179 1996.

${ }^{9}$ Moult, J. The current state of the art in protein structure prediction, Current Opinion in Biotechnology 7: 422-427, 1996.

${ }^{10}$ Seno, F. , Maritan, A. and Banavar, J.R. Interaction potentials for protein folding, Proteins: Structures, Function and Genetics, 30: 244-248, 1988.

${ }^{11}$ Sippl, M.J. Calculation of conformational ensembles from potentials of mean force: an approach to the knowledge based prediction of local structures in globular proteins, J. Mol. Biol., 213: 859-883, 1990.

12 Sippl, M.J.: Knowledge based potentials for proteins, Current Opinion in Structural Biology, 5: 229-235, 1995 and references therein.

13 Tanaka, S. and Scheraga, H.A. Medium and long range interaction parameters between amino acids for predicting threedimensional structures of proteins, Macromolecules 9: 945-950, 1976.

14 Thomas, P.D, and Dill, K. An iterative method for extracting energy like quantities from protein structure, Proc. Natl. Acad. Sci. USA, 93: 11628-11633, 1996.

15 Thomas, P.D. and Dill, K. Statistical potentials extracted from protein structures: how accurate are they? J. Mol. Biol., 257: 457-469, 1996.

${ }^{16}$ Lazaridis, T. and Karplus, M. Effective energy functions for protein structure prediction Curr. Op. in Struct. Biol., 10: 139-145, 2000.

17 Anfinsen, C. Principles that govern the folding of protein chains, Science, 181: 223-230, 1973. 
${ }^{18}$ Bowie, J.U., Luthy, R. and Eisenberg, D. A method to identify protein sequences that fold into a known 3-dimensional structure, Science, 253: 164-170, 1991.

${ }^{19}$ Deutsch, J.M. and Kurosky, T. New algorithm for protein design, Phys. Rev. Lett., 76: 323-326, 1996.

${ }^{20}$ Giugliarelli, G., Micheletti C., Banavar J.R. and Maritan, A., Compactness, aggregation, and prionlike behavior of protein: A lattice model study J. Chem. Phys. 113:5072-5077, 2000.

${ }^{21}$ Micheletti, C., Seno, F., Maritan, A. and Banavar, J.R. Protein design in a lattice model of Hydrophobic and polar amino acids, Phys. Rev. Lett., 80: 2237-2240, 1998.

${ }^{22}$ Micheletti, C., Seno, F., Maritan, A. and Banavar, J.R. Design of proteins with hydrophobic and polar amino acids, Proteins: Structure Function and Genetics, 32: 80-87, 1998.

${ }^{23}$ Micheletti, C. , Maritan, A and Banavar, J.R. A comparative study of existing and new design techniques for protein models, J. Chem. Phys. 110: 9730-9738, 1999.

${ }^{24}$ Morrissey, M.P. and Shakhnovich, E.I. Design of proteins with selected thermal properties, Folding and Design 1: 391-405, 1996.

${ }^{25}$ Pabo, C. Designing proteins and peptides, Nature, 301: 200, 1983.

${ }^{26}$ Quinn, T.P., Tweedy, N.B. , Williams, R.W., Richardson, J.S. and Richardson, D.C. De-novo design, synthesis and characterization of a beta sandwich protein, Proc. Natl. Acad. Sci USA, 91: 8747-8751, 1994

${ }^{27}$ Seno, F., Vendruscolo, M., Maritan, A. and Banavar, J.R. Optimal protein design procedure, Phys. Rev. Lett., 77: 1901-1904, 1996.

${ }^{28}$ Seno, F., Micheletti, C., Maritan, A. and Banavar, J.R. Variational approach to protein design and extraction of interaction potentials, Phys. Rev. Lett., 81: 2172-2175, 1998.

29 A. G. Street and S. L. Mayo. Computational protein design Structure, 7: R105-109, 1999.

${ }^{30}$ R. Du, A. Y. Grosberg and T. Tanaka. Models of protein interactions: how to choose one. Fold. E Des., 3: 203-211, 1998.

${ }^{31}$ van Mourik, J., Clementi, C., Maritan, A. , Seno, F. and Banavar, J.R. Determination of interaction potentials of amino acids from native protein structure: Tests on simple lattice models. J. Chem. Phys. 110: 10123-10133, 1999.

${ }^{32}$ Vendruscolo M. and Domany E. Pairwise contact potentials are unsuitable for protein folding, J. Chem. Phys. 109:1110111108, 1999.

${ }^{33}$ Jones, D. T., Taylor W. R., and Thorton, J. M. A new approach to protein fold recognition. Nature 358: 86, 1992

${ }^{34} \mathrm{H}$. Li, C. Tang and N. S. Wingreen. Nature of driving force for protein folding; a result from analyzing the statistical potential Phys. Rev. Lett. 79:765-768, 1997.

${ }^{35}$ E. S. Huang, P. Koehl, M. Levitt, R. V. Pappu and J. W. Ponder. Accuracy of side-chain prediction upon near-native protein backbones generated by ab initio foling methods. Proteins: struct. funct. and gen. 33: 204-207, 1998.

${ }^{36}$ Clementi, C., Maritan, A. and Banavar, J.R. Folding, design and determination of interaction potentials using off-lattice dynamics of model heteropolymers, Phys. Rev. Lett., 81: 3287-3290, 1998.

${ }^{37}$ Bryngelson J. D. and Wolynes, P. G. Spin glasses and the statistical mechanics of protein folding, Proc. Natl. Acad. Sci. USA 84: 7524-7528, 1987.

38 J. D. Bryngelson and P. G. Wolynes, Proc. Nat. Acad. Sci. USA, 84, 7524 (1987); P. G. Wolynes, J. N. Onuchic and D. Thirumalai, Science 267, 1619 (1995); K. A. Dill and H. S. Chan, Nature Structural Biology 4, 10 (1997).

${ }^{39}$ C. Micheletti, J. R. Banavar, A. Maritan and F. Seno, Protein structures and optimal folding from a geometrical variational principle Phys. Rev. Lett. 82, 3372-3375, 1999.

${ }^{40}$ D. A. Debe, M. J. Carlson and W. A. Goddard. The topomer-sampling model of protein folding Proc. Natl. Acad. Sci. USA, 96: 2596-2601, 1999.

${ }^{41}$ Park, B.H. and Levitt, M. The complexity and accuracy of discrete state models of protein structure, J. Mol. Biol., 249: 493-507, 1995.

42 Micheletti, C. , Seno, F. Maritan, A and Banavar, J.R. Strategies for protein folding and design. Annals of Combinatorics, 3: 439-458, 1999.

${ }^{43}$ Covell, D. G. and Jernigan, R. Conformations of folded proteins in restricted spaces. Biochemistry 29: 3287, 1990.

${ }^{44}$ Park, B.H. and Levitt, M. Energy functions that discriminate X-ray and near-native folds from well-constructed decoys. $J$. Mol. Biol. 258: 367-392, 1996.

${ }^{45}$ Krauth, W. \& Mezard, M. Learning algorithms with optimal stability in neural networks. J. Phys. A. 20: L745-L752, 1987.

${ }^{46}$ Clementi, C. "Effective Potentials for Protein Folding Models", Ph. D. Thesis, SISSA, Trieste, 1998. 


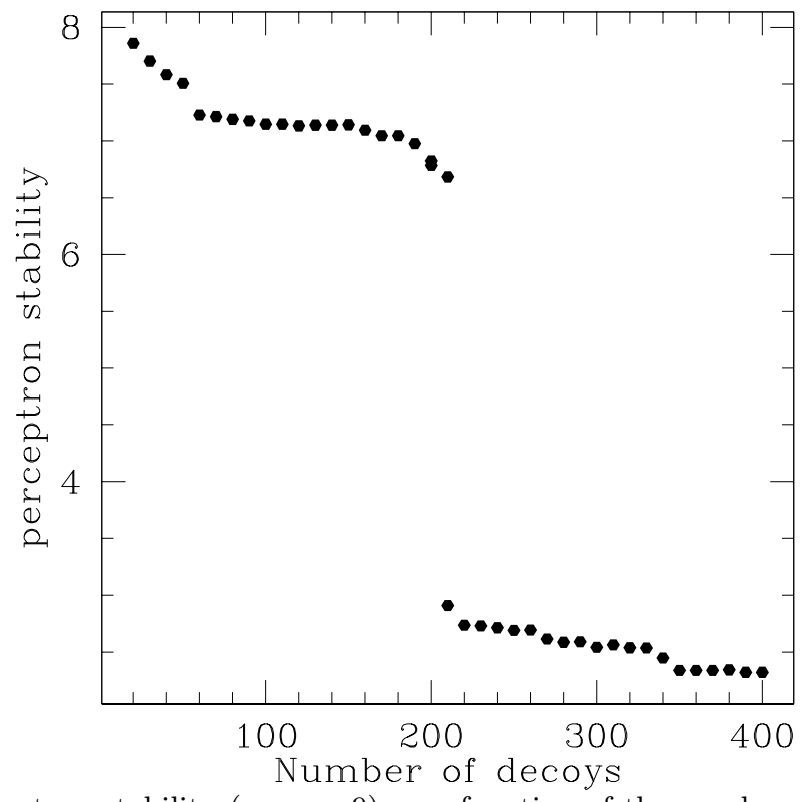

FIG. 1. Behaviour of the perceptron stability (see eq. 9) as a function of the number of decoys generated for protein 1vcc with model A. The first 100 decoys were generated from an initial random set of potentials. Then, the potentials were refined to provide maximum stability. When the new set of potentials are used to extract other 100 decoys, a dramatic increase in thermodynamic stability is observed. The same effect is repeated by iterating the potential learning procedure and generating other 100 decoys This proves the effectiveness of updating the potentials by the appearance of more stringent decoys than the initial ones.
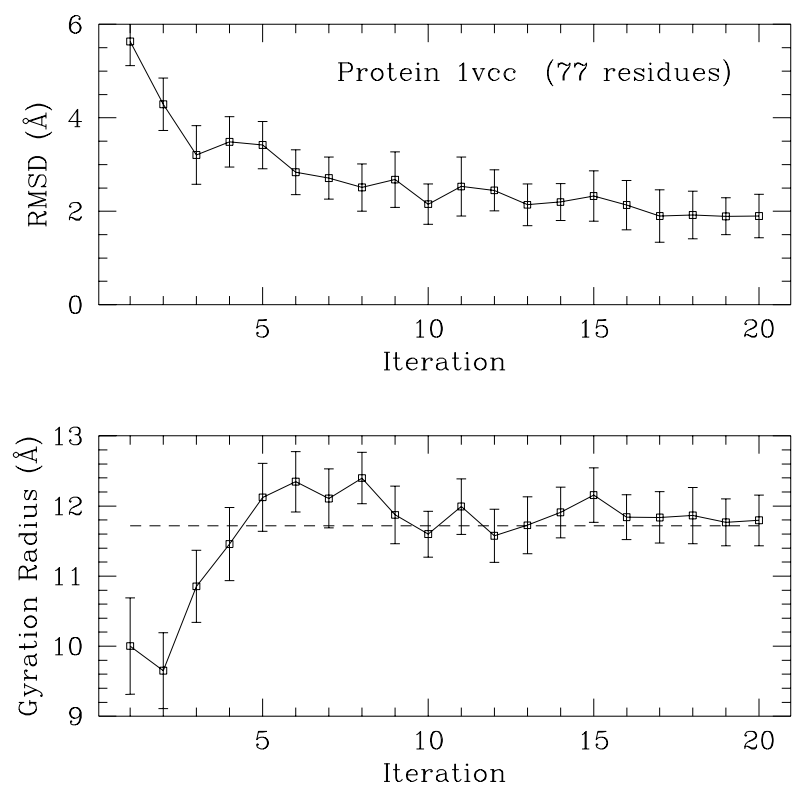

FIG. 2. Results of the iterations on the single protein 1vcc. In the upper plot we show the asymptotic RMSD of the decoy structures as a function of iteration (potential updates). Each point represents an average over 30 decoys. In the lower plot we show the corresponding radius of gyration of the asymptotic decoys. The dashed line shows the gyration radius of the native structure of 1vcc. 


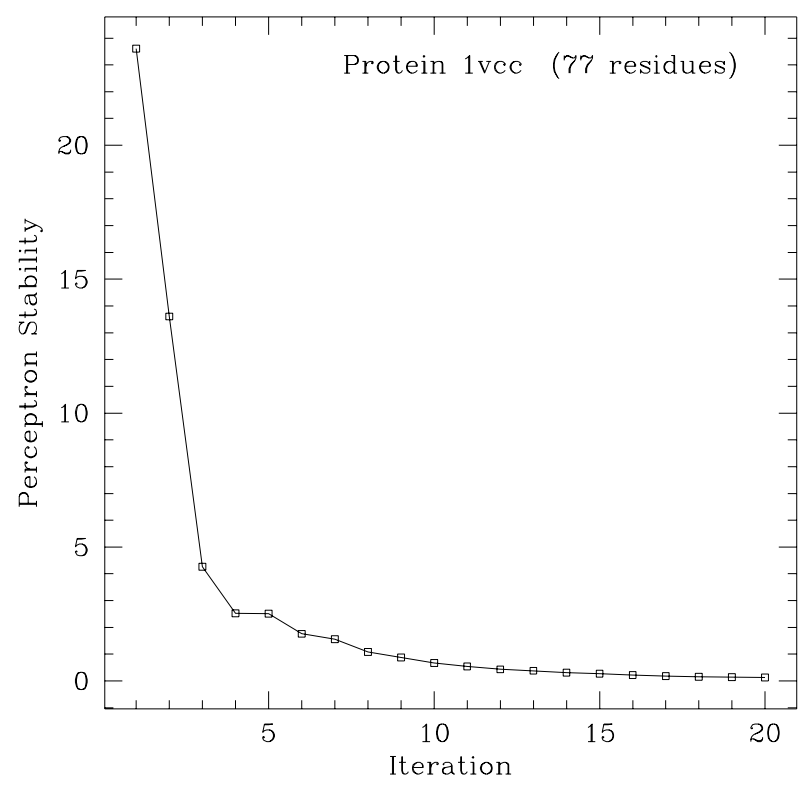

FIG. 3. This plot shows how the perceptron stability decreases as the iterations proceed on the single protein 1vcc. Extrap olating to an infinite number of iterations, one obtains negative values for the stability, consistent with previous observation 32 that pairwise potentials are insufficient to stabilize the native state. Nevertheless, we show that a single protein can be stabilized within a narrow native basin (see previous figure).
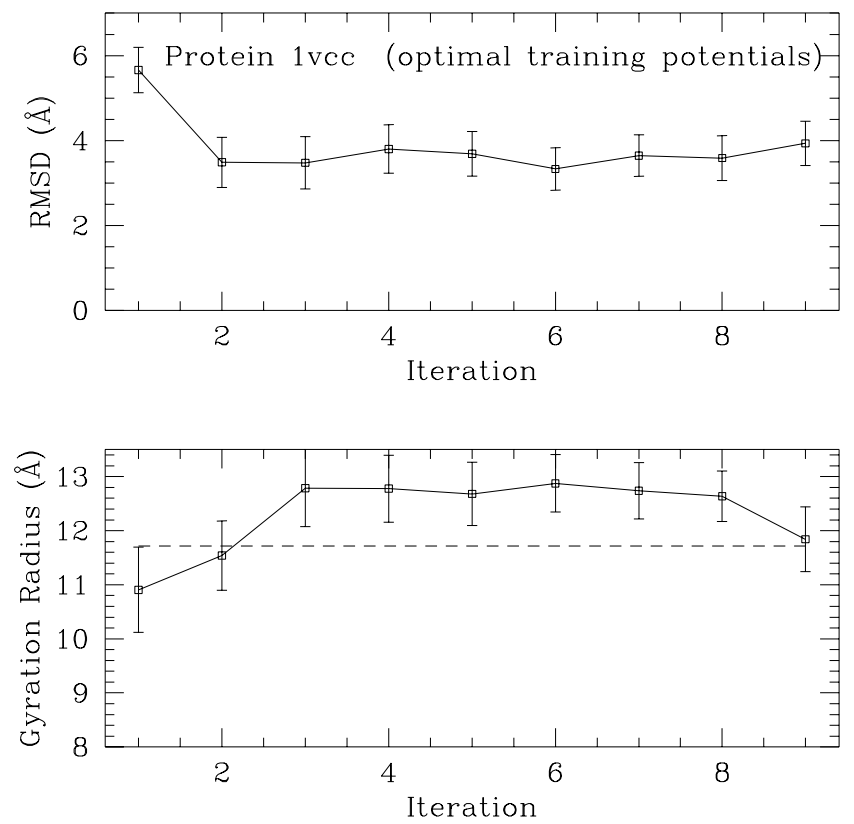

FIG. 4. Asymptotic RMSD and radius of gyration of the decoy structures obtained with the protein 1vcc during the iteration procedure of the ensemble of 20 proteins in Table At each iteration stage, 5 decoys are generated and the averages and the fluctuations are calculated with these 5 decoys. 


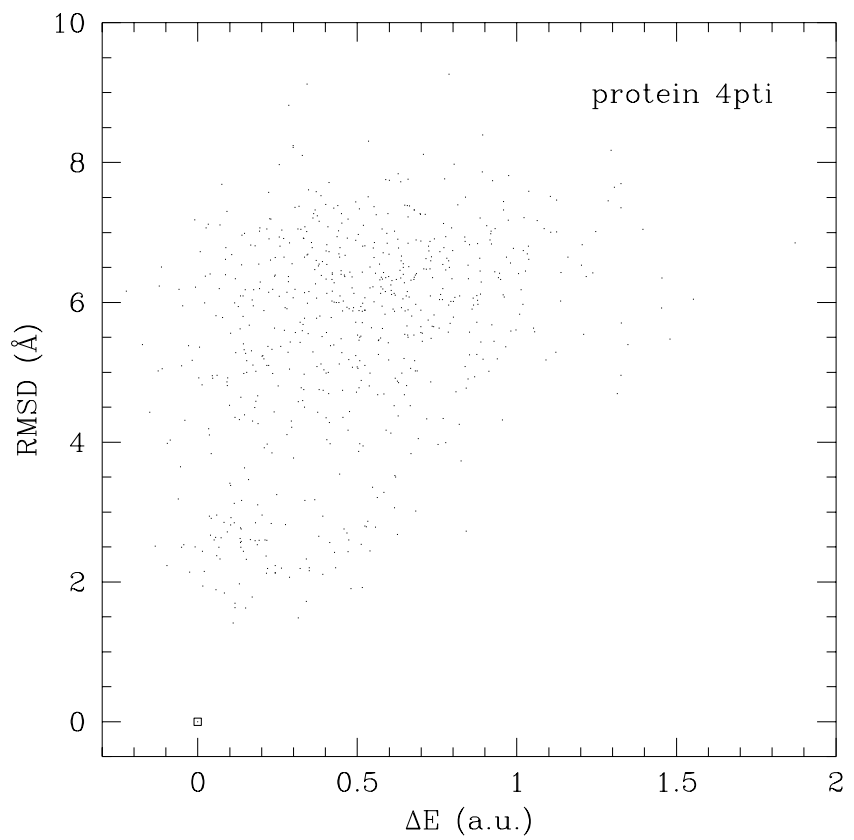

FIG. 5. Scatter plot of the rmsd vs energy gap (in abritrary units) for the Park and Levitt decoys (there are more than 600 decoys) for protein 4pti. The energy gap (defined as the difference in energy between the decoy energy and the true native state energy) was calculated using the optimal potentials deduced from the stabilization of the 20 proteins in the training set. Notice that the decoys of Park and Levitt (and the native state used for comparing the energy) were not constrained to be on the FCC lattice.

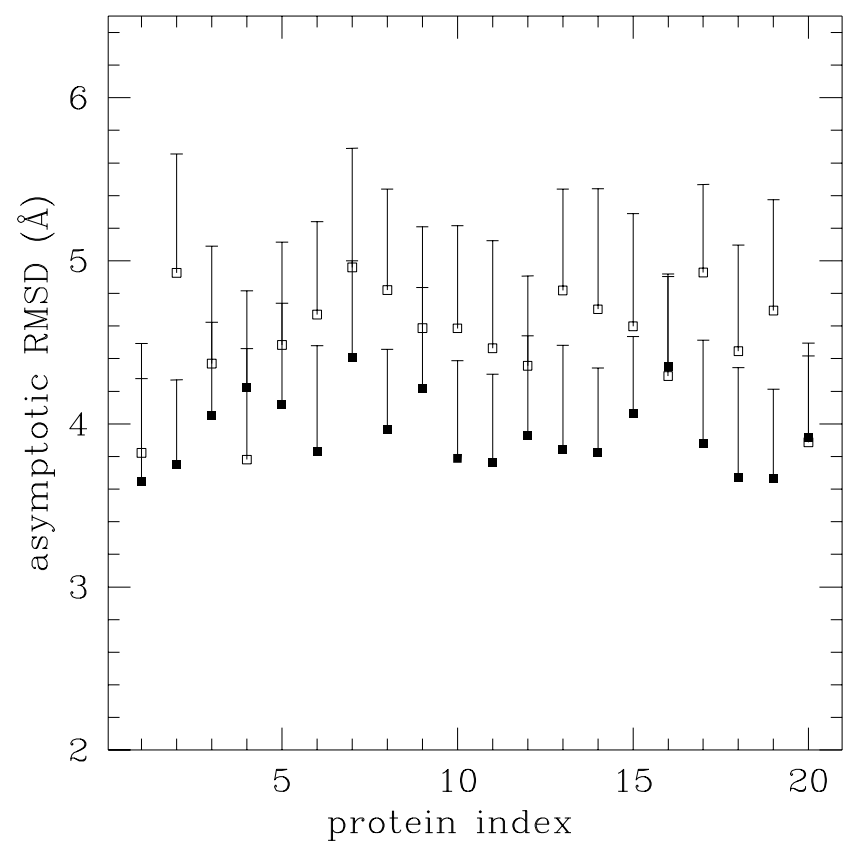

FIG. 6. Average asymptotic RMSD obtained obtained for each of the twenty proteins of the training set with our potentials (filled squares) and with those obtained by learning the Park and Levitt decoys (open squares). Averages and fluctuations are calculated with 5 decoys determined by asymptotically relaxing the native state under the action of the Monte Carlo dynamics. Errorbars are shown only on one side of the points, to avoid confusing overlaps. 


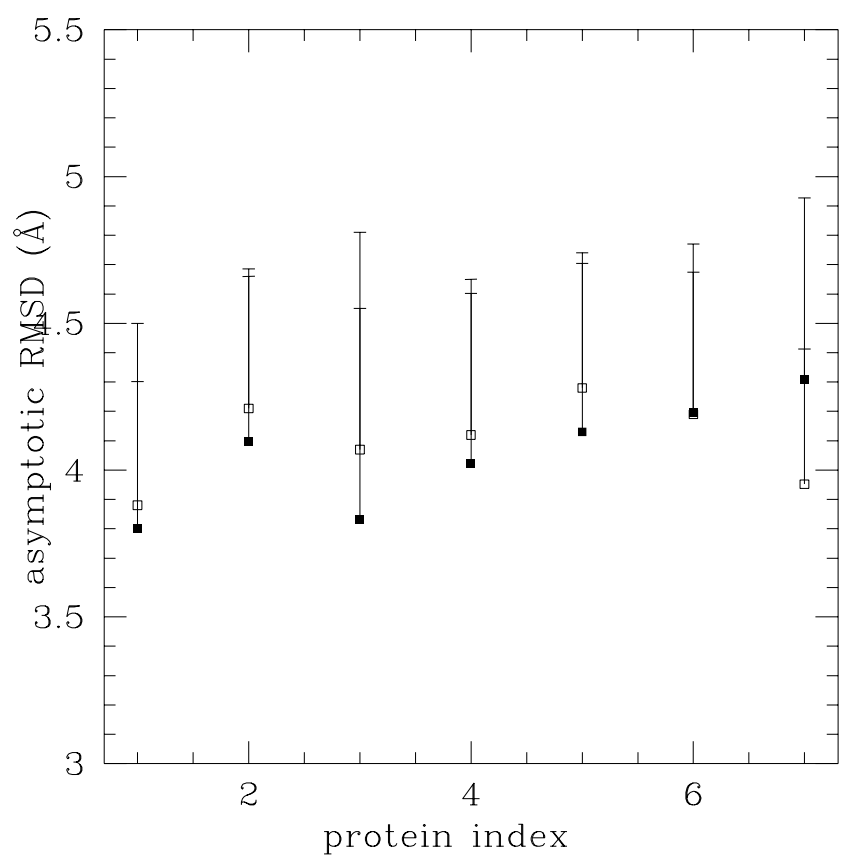

FIG. 7. Average asymptotic RMSD obtained for each of the seven Park andLevitt proteins with our potentials (filled squares) and with those obtained by learning the Levitt decoys (open squares) Averages and fluctuations are calculated with 5 decoys determined by asymptotically relaxing the native state under the action of the Monte Carlo dynamics. Errorbars are shown only on one side of the points, to avoid confusing overlaps.

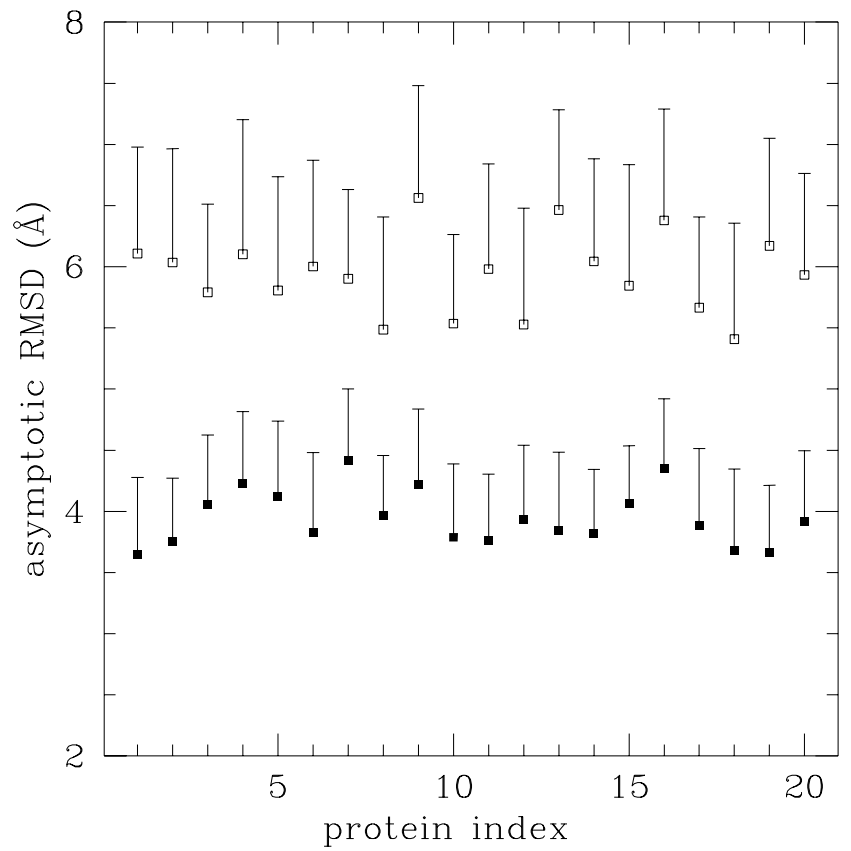

FIG. 8. Average asymptotic RMSD obtained for each of the twenty proteins of the training set with our potentials (filled squares) and with those obtained using the quasichemical approach (open squares). Averages and fluctuations are calculated with 5 decoys determined by asymptotically relaxing the native state under the action of the Monte Carlo dynamics. Errorbars are shown only on one side of the points, to avoid confusing overlaps. 


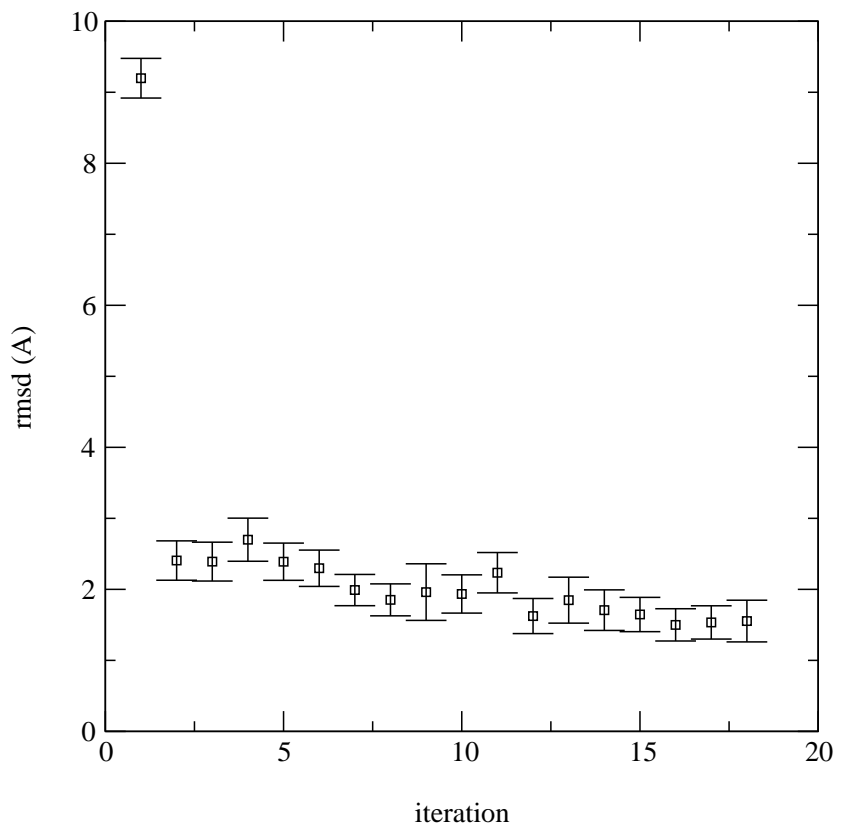

FIG. 9. Results of the iterations on the single protein 1vcc obtained with the more sophisticated model B described in text. The asymptotic RMSD of the decoy structures is shown as a function of iteration (potential updates). Each point represents an average over 30 decoys. In this case the value of RMSD is bigger at the first iteration because, in this model, the $C_{\alpha}-C_{\alpha}$ and $C_{\alpha}-C_{\beta}$ hard core repulsions are removed and substituted by a potential that is learned during the iterative procedure. Initially, this potential is 0 and the resulting conformations can be extremely compact. 


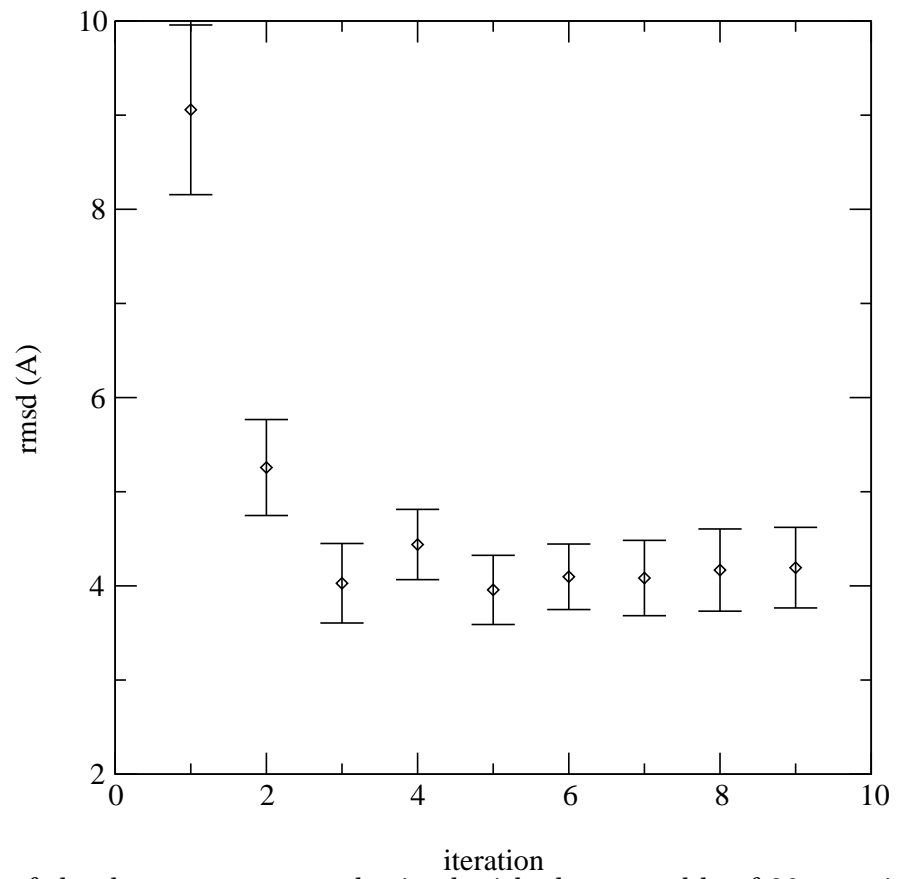

FIG. 10. Asymptotic RMSD of the decoy structures obtained with the ensemble of 20 proteins in Table I with the model B. At each iteration stage, 5 decoys per protein are generated and the averages and the fluctuations are calculated with these 100 decoys. 


\begin{tabular}{|c|c|}
\hline Protein & Length| \\
\hline $1 \mathrm{cbn}$ & 46 \\
\hline 1 coo & 81 \\
\hline $1 \mathrm{erv}$ & 105 \\
\hline $1 \mathrm{fna}$ & 91 \\
\hline 1 fow & 76 \\
\hline 1hoe & 74 \\
\hline 1hyp & 75 \\
\hline $1 \mathrm{kjs}$ & 74 \\
\hline $1 \mathrm{mit}$ & 69 \\
\hline 1opd & 85 \\
\hline 1pdo & 129 \\
\hline 1rro & 108 \\
\hline 1sap & 66 \\
\hline 1 shg & 57 \\
\hline 1tif & 76 \\
\hline 1ubi & 76 \\
\hline 1utg & 70 \\
\hline $1 \mathrm{vcc}$ & 77 \\
\hline $2 \mathrm{fxb}$ & 81 \\
\hline $2 \mathrm{imm}$ & 114 \\
\hline
\end{tabular}

TABLE I. The trial set of 20 single-chain proteins used for extracting the interaction potentials between amino acids. The set includes proteins representative of the most common folds and lengths.

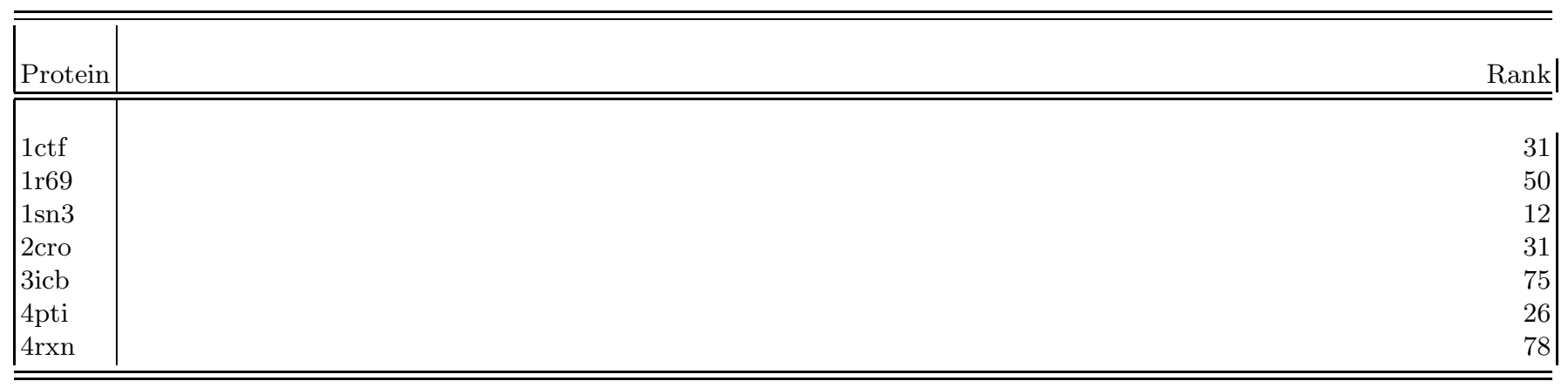

TABLE II. Ranking (using model A) of the 7 native proteins for which more than 600 highly competitive decoys have been obtained by Park and Levitt. The ranking is measured by comparing the native energy (the optimal potentials were the ones obtained using the 20 proteins in the training set) with those of the decoys. 


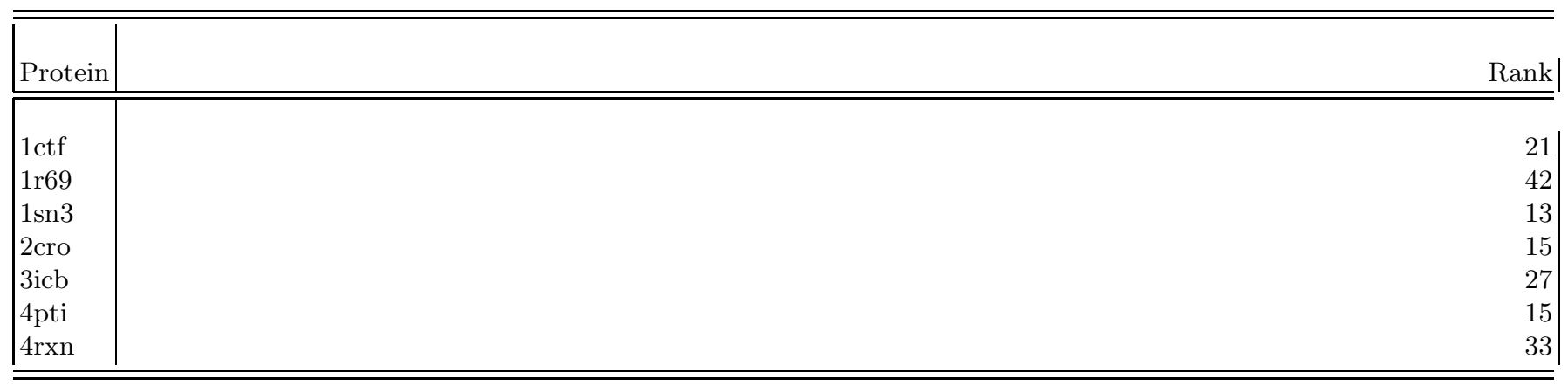

TABLE III. Ranking (using model B) of the 7 native proteins for which more than 600 highly-competing decoys have been obtained by Park and Levitt. 


\section{APPENDIX A: TABLE OF OPTIMAL INTERACTIONS}

Below is included the table of the optimal interactions extracted with Model A. The corresponding value for $\epsilon_{r}$ is 0.032594 .

\begin{tabular}{|c|c|c|c|c|c|c|c|c|c|c|c|}
\hline GLY & "GLY & 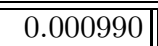 & VAL & $\overline{\mathrm{ASP}}$ & 0.000092 & MET & PHE & 0.001010 & 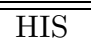 & 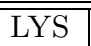 & 0.002934 \\
\hline GLY & ALA & -0.003111 & VAL & ASN & -0.001040 & MET & PRO & -0.008698 & HIS & $\mathrm{ARG}$ & 0.009985 \\
\hline GLY & VAL & 0.001995 & VAL & GLU & 0.001387 & MET & TYR & -0.003258 & HIS & $\mathrm{ASP}$ & -0.002501 \\
\hline GLY & LEU & -0.001538 & VAL & GLN & 0.000029 & MET & HIS & 0.031785 & HIS & ASN & 0.008099 \\
\hline GLY & ILE & 0.000446 & LEU & LEU & -0.000748 & MET & $\mathrm{TRP}$ & 0.984886 & HIS & GLU & -0.007232 \\
\hline GLY & CYS & 0.001847 & $\mathrm{LEU}$ & ILE & -0.000782 & MET & SER & 0.002007 & HIS & GLN & 0.005803 \\
\hline GLY & MET & 0.002339 & LEU & CYS & -0.000196 & MET & THR & -0.002190 & TRP & TRP & 0.131813 \\
\hline GLY & TYR & -0.000737 & LEU & PRO & -0.005026 & MET & ASP & 0.001491 & TRP & LYS & -0.001668 \\
\hline GLY & HIS & -0.000951 & LEU & TYR & 0.003540 & MET & $\mathrm{ASN}$ & 0.018413 & TRP & $\mathrm{ARG}$ & -0.014845 \\
\hline GLY & TRP & -0.012366 & LEU & HIS & -0.004529 & MET & GLU & 0.003231 & TRP & $\mathrm{ASP}$ & -0.007832 \\
\hline GLY & SER & -0.003528 & LEU & TRP & 0.010659 & MET & GLN & -0.002908 & TRP & $\mathrm{ASN}$ & -0.003028 \\
\hline GLY & THR & 0.001084 & LEU & SER & 0.001004 & PHE & PHE & -0.013128 & TRP & GLU & -0.009357 \\
\hline GLY & LYS & -0.001308 & LEU & THR & 0.003770 & PHE & $\mathrm{PRO}$ & -0.006986 & TRP & GLN & 0.012075 \\
\hline GLY & GLN & -0.000425 & LEU & GLU & -0.000453 & PHE & THR & 0.004102 & SER & ASP & -0.001609 \\
\hline ALA & ALA & 0.001461 & LEU & GLN & -0.004168 & PHE & LYS & -0.004479 & SER & $\mathrm{ASN}$ & 0.006249 \\
\hline ALA & VAL & 0.003642 & ILE & ILE & 0.006801 & PHE & $\mathrm{ARG}$ & 0.004855 & SER & GLU & 0.002888 \\
\hline ALA & LEU & 0.000864 & ILE & CYS & 0.002965 & PHE & $\mathrm{ASP}$ & 0.004899 & SER & GLN & -0.009002 \\
\hline ALA & ILE & -0.002119 & ILE & MET & -0.009283 & PHE & $\mathrm{ASN}$ & 0.003461 & THR & THR & 0.003269 \\
\hline ALA & CYS & -0.000751 & ILE & PHE & -0.009792 & PHE & GLU & -0.001143 & THR & LYS & -0.005895 \\
\hline ALA & MET & -0.001496 & ILE & PRO & 0.004353 & PHE & GLN & 0.003790 & THR & $\mathrm{ARG}$ & 0.003967 \\
\hline ALA & PHE & 0.005126 & ILE & TYR & -0.004792 & PRO & $\mathrm{PRO}$ & -0.003621 & THR & ASP & 0.002193 \\
\hline ALA & PRO & -0.005081 & ILE & HIS & -0.000476 & PRO & TYR & 0.000996 & THR & $\mathrm{ASN}$ & -0.005914 \\
\hline ALA & TYR & -0.000724 & ILE & TRP & 0.002734 & PRO & HIS & -0.002032 & THR & GLU & 0.000948 \\
\hline ALA & HIS & -0.002432 & ILE & SER & 0.001538 & PRO & $\mathrm{TRP}$ & 0.013914 & THR & GLN & 0.001006 \\
\hline ALA & GLU & -0.002376 & CYS & CYS & -0.002544 & PRO & GLN & 0.000525 & $\mathrm{ARG}$ & ASP & 0.001974 \\
\hline ALA & GLN & 0.005029 & CYS & MET & 0.014331 & TYR & TYR & -0.007699 & ARG & $\mathrm{ASN}$ & -0.006728 \\
\hline VAL & VAL & 0.001445 & CYS & PHE & -0.013925 & TYR & HIS & 0.007276 & $\mathrm{ARG}$ & GLU & -0.004586 \\
\hline VAL & LEU & -0.001940 & CYS & $\mathrm{PRO}$ & -0.001720 & TYR & $\mathrm{TRP}$ & 0.003708 & ARG & GLN & -0.001210 \\
\hline VAL & ILE & 0.002618 & CYS & TYR & 0.002585 & TYR & SER & -0.001895 & ASP & ASP & -0.000531 \\
\hline VAL & CYS & 0.000296 & CYS & HIS & 0.054553 & TYR & THR & -0.001235 & ASP & $\mathrm{ASN}$ & 0.007855 \\
\hline VAL & MET & -0.005331 & CYS & TRP & -0.035239 & TYR & LYS & 0.007956 & ASP & GLU & 0.002194 \\
\hline VAL & PHE & 0.000008 & CYS & SER & 0.001837 & TYR & $\mathrm{ARG}$ & 0.004237 & ASP & GLN & -0.001466 \\
\hline VAL & PRO & 0.001362 & CYS & THR & 0.002620 & TYR & $\mathrm{ASP}$ & 0.000182 & ASN & $\mathrm{ASN}$ & -0.001962 \\
\hline VAL & TYR & -0.002175 & CYS & LYS & -0.006040 & TYR & ASN & -0.006968 & ASN & GLU & -0.003154 \\
\hline VAL & HIS & -0.006893 & CYS & $\mathrm{ARG}$ & -0.006062 & TYR & GLU & 0.003261 & ASN & GLN & 0.004502 \\
\hline VAL & $\mathrm{TRP}$ & -0.001516 & CYS & ASP & 0.002278 & TYR & GLN & -0.005137 & GLU & GLU & 0.006456 \\
\hline VAL & SER & -0.000443 & CYS & $\mathrm{ASN}$ & 0.006139 & HIS & HIS & 0.001314 & GLU & GLN & -0.005234 \\
\hline VAL & THR & 0.004075 & CYS & GLU & 0.002791 & HIS & TRP & -0.006739 & GLN & GLN & 0.008438 \\
\hline VAL & LYS & -0.006987 & CYS & GLN & 0.001387 & HIS & SER & 0.009858 & & & \\
\hline VAL & $\mathrm{ARG}$ & -0.005168 & MET & MET & 0.031655 & HIS & THR & -0.005871 & & & \\
\hline
\end{tabular}

